\title{
HAUPTBEITRĀGE \\ Der Capability-Ansatz in der Gesundheitsförderung: Ansatzpunkte für eine Neuausrichtung der Ungleichheitsforschung
}

$10 \ddot{z} \mathrm{~S}_{-}$

\author{
Thomas Abel $\cdot$ Dominik Schori
}

Zusammenfassung: Gesundheitliches Wohlergehen geht unmittelbar auf das aktive Zutun von Personen und Kollektiven zurück. Zugleich wird gesundheitsrelevantes Handeln nur unter der Berücksichtigung der jeweiligen physischen und sozialen Kontexte zu verstehen und zu beeinflussen sein. Dementsprechend wird hier eine Ausrichtung der modernen Gesundheitsförderung auf die gesundheitsrelevanten Ressourcen und Handlungsspielräume der Menschen vorgeschlagen. Hierfür sind theoretische Grundlagen erforderlich, die die Fragen der sozialen Ungleichheit mit den praktischen Zugängen des Empowerment und der Partizipation schlüssig verbinden. Die Autoren stellen dazu den Capability-Approach (CA) von Amartya Sen vor und ergänzen diesen mit Erkenntnissen aus der Kapital-Interaktionstheorie von Pierre Bourdieu. Beide Ansätze beleuchten Grundfragen der sozialen Ungleichheit und können mit den Leitkonzepten der Ottawa-Charta fruchtbar verbunden werden. Sie liefern damit auch Anleitungen für neue Forschungsrichtungen zur Untersuchung der komplexen Wechselwirkungen von sozialen Kontexten und gesundheitsrelevantem Handeln.

Schlüsselwörter: Capability-Ansatz · Gesundheitsforschung · Soziale Ungleichheit · Kapitalformen $\cdot$ Lebensstile

\section{The Capability Approach in Health Promotion: Towards a Re-orientation of Inequality Research}

Abstract: Health-related well-being is a result of individual or collective agency. Understanding and influencing health-relevant behaviour, however, requires to account for physical and social contexts of agency. Accordingly, the authors suggest a focus in modern health promotion on people's health-relevant resources and capabilities. This requires a theoretical basis onto which issues of social inequality can be linked to specific approaches of health promotion practice, namely empowerment and participation. To this purpose, the authors present Amartya Sen's theory-based Capability Approach (CA) and complement it with insights from Pierre Bourdieu's theory of capi-

Prof. Dr. Thomas Abel, PhD. (ه) Department of Social and Preventive Medicine Universität Bern Niesenweg 6 3012 Bern, Schweiz E-Mail: abel@ispm.unibe.ch
Dominik Schori $(\varangle)$

Department of Social and

Preventive Medicine

Universität Bern

Niesenweg 6

3012 Bern, Schweiz

E-Mail: dschori@ispm.unibe.ch 
tal interaction. Both of these theories shed light on key issues of social inequality and can fruitfully be associated with the guiding principles in health promotion stipulated by the Ottawa Charter. Together, they provide guidelines for new areas of research to analyse the complex interplay between health behaviour and social context.

Keywords: Capability Approach - Health Promotion Research - Social Inequality - Capitals · Lifestyles

\section{Einleitung}

\subsection{Gesundheitliche Ungleichheit, sozialer Kontext und Gesundheitsförderung}

Die Zusammenhänge von sozialer und gesundheitlicher Ungleichheit sind heute in eindrücklichem Maß statistisch belegt (Mackenbach 2006; Siegrist und Marmot 2008). Niedrigeres Einkommen und geringere Schulbildung sind mit höheren Wahrscheinlichkeiten von Erkrankungen, häufigerem Auftreten von gesundheitlichen Risiken und geringerer durchschnittlicher Lebenserwartung assoziiert. Dies gilt für fast alle Länder, in denen verlässliche epidemiologische Daten vorliegen, einschließlich dem deutschsprachigen Raum (Helmert, Bammann und Müller 2000; Bisig, Bopp und Minder 2001; Lampert et al. 2005; Richter 2005; Spoerri et al. 2006).

Neben der unterschiedlichen Risikoexposition (z. B. je nach Berufsgruppen) und möglichen Unterschieden in der Gesundheitsversorgung (z. B. Zugang zu medizinischen Versorgungsmaßnahmen) werden zur Erklärung dieser Variationen die Einflüsse unterschiedlicher Lebensweisen auf die Gesundheit untersucht (vgl. im Überblick Mielck 2005; Richter und Hurrelmann 2006).

Gesundheitsförderliches und gesundheitsabträgliches Verhalten zeigt sich in empirischen Studien häufig mit sozialen Merkmalen wie Bildung, Einkommen und Berufsstatus assoziiert (Blaxter 1990; Droomers, Schrijvers und Mackenbach 2001; Thrane 2006; Kouvonen et al. 2007; Currie et al. 2008).

Solche Ergebnisse weisen auf die Bedeutung von spezifischen Verhaltens- und breiteren Handlungsmustern für die Erklärung der Sozialschichtsunterschiede in Gesundheit und Krankheit hin. Der Ansatz der Gesundheitsförderung trägt der Perspektive, dass Gesundheit im alltäglichen Handeln von den Individuen selbst mitgcschaffen bzw. erhalten wird (z. B. über präferierte Konsumgewohnheiten, Freizeitgestaltungen etc.), in besonderem Maße Rechnung. So heißt es in der Ottawa-Charta (1986), dem Gründungsmanifest der modernen Gesundheitsförderung, dass Gesundheit dadurch entstehe, ,[. . .] dass man sich um sich selbst und für andere sorgt, dass man in die Lage versetzt ist, selber Entscheidungen zu fällen und eine Kontrolle über die eigenen Lebensumstände auszuüben [.. .] (Ottawa-Charta 1986).“

In der Perspektive der modernen Gesundheitsförderung wird Gesundheit selbst zudem nicht auf einen Status des ,Nicht-erkrankt-Seins" reduziert, sondern verstanden als umfassendes körperliches, seelisches und soziales Wohlbefinden. Dabei kommt gesundheitliches Wohlbefinden zu Stande, indem Menschen ,[. . .] ihre Bedürfnisse befriedigen, ihre Wünsche und Hoffnungen wahrnehmen und verwirklichen sowie ihre Umwelt meistern bzw. verändern können (ebd). “ 
Verhaltensbezogene Ansätze (wie z. B. mehr gesunde Bewegung im Alltag, ausgeglichene Ernährung) machen heute einen Großteil der Interventionen in der Gesundheitsförderung aus. Dabei sind jedoch wichtige grundlegende Fragen zur sozialen Ungleichheit offen. So wissen wir bis heute wenig über die Kontextgebundenheit von gesundheitlichem Handeln und zur relativen Bedeutung von finanziellen, sozialen und kulturellen Ressourcen für gesundheitsförderliche Lebensstile innerhalb verschiedener Bevölkerungsschichten (u. a. Frohlich, Corin und Potvin 2001; Abel 2007), was auch mit den mangelnden theoretischen Grundlagen der Gesundheitsförderung erklärt werden kann (McQueen und Anderson 2001).

Das Paradigma der Gesundheitsförderung konkretisiert sich vorwiegend über praktische Zugänge und Prozesse der Gesundheitserhaltung und -entstehung: Empowerment und Partizipation sind in der Gesundheitsförderung tragende Elemente eines Prozess, der ,[. . . ] allen Menschen ein höheres Maß an Selbstbestimmung über ihre Gesundheit ermöglichen und sie damit zur Stärkung ihrer Gesundheit befähigen" soll (OttawaCharta 1986). Dies wird letztlich jedoch nur dadurch möglich, dass „[. . .] die Gesellschaft, in der man lebt, Bedingungen herstellt, die all ihren Bürgern Gesundheit ermöglichen (ebd). " Damit betont der Ansatz die Bedeutung der Lebensverhältnisse in gleichem $\mathrm{Ma} ß$ wie die der Verhaltenseinflüsse auf Gesundheit.

Definiert als umfassendes Wohlbefinden und erklärt als das Resultat des Zusammenspiels von Verhalten und Verhältnissen, öffnet sich das Thema Gesundheit für die soziologische Theorie: So hat die Anwendung von Max Webers Erkenntnissen über die duale Struktur der Lebensstile geholfen zu verstehen, wie das Zusammenspiel von Lebensführung und strukturell verankerten Lebenschancen die Entstehung von gesundheitsrelevanten Lebensstilen bestimmt (Abel 1991; Cockerham, Abel und Lüschen 1993). Pierre Bourdieus Konzept des Habitus liefert darüber hinaus eine theoretisch fundierte Perspektive, die in empirischen Arbeiten der Gesundheitsforschung erfolgreich eingesetzt worden ist (Williams 1995; Cockerham, Rütten und Abel 1997; Abel, Cockerham und Niemann 2000; Sperlich und Mielck 2003; Veenstra 2007).

\subsection{Entscheidungen für gesundheitsförderliches Verhalten im sozialen Kontext}

Während sich auch unter den Bedingungen moderner Geseilschaften gesundheitsrelevante Verhaltensweisen nach wie vor als schichtabhängige Determinanten der Gesundheitschancen zeigen, lassen sich zugleich auch grundlegende Veränderungen in den Anforderungen an einzelne Personen konstatieren. Die Gestaltung von Lebensstilen umfasst heute mehr denn je immer auch Entscheidungen für oder gegen spezifische und oftmals gesundheitsrelevante Verhaltensweisen. Mehr noch: Die Notwendigkeit, sich tagtäglich für oder gegen spezifische Verhaltensweisen zu entscheiden, scheint in modernen Gesellschaften zuzunehmen. Unter den „Bedingungen der Moderne“, argumentiert Anthony Giddens, müssen Menschen in einem Umfeld eines wachsenden Angebotes an Informationen, Konsumgütern und Dienstleistungen immer häufiger aktive Entscheidungen treffen. Individuen haben ,no choice but to choose“ (Giddens 1991:81) und sind auch hinsichtlich der individuellen Risikoabschätzung ihrer Handlungen und Zustände zunehmend auf eine reflexive Aneignung von Wissen angewiesen (Beck 1986; Giddens 
1991). In diesem Sinne müssen inbesondere die verhaltensbezogenen Ansätze der Gesundheitsförderung den Bedingungen Rechnung tragen, welche es Individuen ermöglichen, gesundheitsförderliche Entscheidungen zu treffen und gesundheitsförderliches Verhalten umzusetzen.

Damit rückt das Thema der sozialen Ungleichheit und der ungleichen Handlungsspielräume (z. B. für gesunde Lebensstile) in das Blickfeld der Gesundheitsförderung. Bisherige Forschungsarbeiten liefern dazu kaum Ergebnisse. Sozialepidemiologische Studien bieten meist deskriptive Erkenntnisse zu den statistischen Zusammenhängen von sozialer Ungleichheit und gesundheitlichen Konsequenzen, wie z. B. der Erkrankungshäufigkeit. Solche Erkenntnisse - wiewohl beispielsweise für die medizinische Versorgungsplanung äußerst wertvoll - entsprechen jedoch kaum der Ausrichtung der modernen Gesundheitsförderung. Wir gehen hier davon aus, dass die spezifische Ausrichtung der Gesundheitsförderung auf der Basis ihrer Leitprinzipien Partizipation und Empowerment es erfordert, die Grundfragen der sozialen Ungleichheit neu zu stellen.

Der Ausgangspunkt der Gesundheitsförderung liegt bei einem Begriff der Gesundheit als positiv definierte Größe (z. B. Lebensqualität und Wohlbefinden) und bei den sozialen Bedingungen gesunder Entwicklung von Menschen als social agents, die in ihrem Handeln nicht nur von sozialen Strukturen abhängig sind, sondern diese immer auch selber prägen (Abel, Cockerham und Niemann 2000; Cockerham 2005). Entscheidend für die Gestaltungsmöglichkeiten ist dabei die Ausstattung mit materiellen und nichtmateriellen Ressourcen. In diesem Zusammenhang verstehen wir Ressourcen als Mittel zu dem Zweck, etwas als erstrebenswert Erachtetes zu erreichen. Dem Grundsatzgedanken der Gesundheitsförderung zum Empowerment folgend, muss dabei das Ziel vom Individuum selbst bestimmt sein. Wenn eine Entscheidung für gesundheitsförderliches Handeln gefallen ist - sei dies nun das eigene alltägliche Bewegungsverhalten oder das auf Gesundheit ausgerichtete Engagement in einer Gemeinde -, müssen die entsprechenden Umsetzungsmöglichkeiten gegeben sein. Die Hypothese liegt nahe, dass diese Umsetzungsmöglichkeiten auch im Bereich der gesundheitsrelevanten Entscheidungen und Lebensentwürfe von der Ausstattung mit spezifischen Ressourcen abhängen.

Zukünftig braucht die Gesundheitsförderung spezifische theoretische Anleitungen, die ihrer Zielsetzung zur Schaffung von mehr Chancengleichheit entsprechen, dabei über krankheitsbezogene Kriterien hinausgehen und zugleich ihren Leitprinzipien des Empowerment und der Partizipation gerecht werden können. Mizhael Marmot hat unlängst darauf hingewiesen, dass Amartya Sens Capability Approach dazu geeignet ist, gesundheitliche Ungleichheit als Folge eines Mangels an Empowerment und Partizipation zu deuten (Marmot 2006: 2089). Bis jetzt hat jedoch kaum eine breite Diskussion über die theoretischen Implikationen stattgefunden, die eine Anwendung des Capability Approaches (CA) in der Gesundheitsförderung mit sich bringt.

Unser Beitrag möchte zeigen, dass der CA eine tragfähige theoretische Grundlage für die moderne Gesundheitsförderung bietet. Die Anwendung des Capability Approaches führt uns zu einer neuen theoretisch begründeten Zielbestimmung, nach der die Erweiterung der gesundheitsförderlichen Handlungsspielräume als zeitgemäße und praxisorientierte Zielgröße der Gesundheitsförderung definiert werden kann. Auf dieser Grundlage können und müssen spezifische Anforderungen für eine neue Ausrichtung der Ungleichheitsforschung in der Gesundheitsförderung definiert werden. 
Im Hauptteil werden zunächst die wichtigsten Elemente des CA dargestellt ${ }^{1}$. Die spezifische Anwendung dieser Elemente auf die Gesundheitsförderung ermöglichen es, gesundheitsrelevante Handlungsoptionen als ihren zentralen Gegenstand zu bestimmen und konzeptuell einzubinden. In einem zweiten Schritt wird der CA mit der Bourdieu'schen Theorie der ökonomischen, kulturellen und sozialen Kapitalien unterfüttert. Diese Ergänzung erscheint aus der Sicht einer kritischen Gesundheitsförderung erforderlich, um speziell die Bedeutung sozial ungleich verteilter Ressourcen bei der Entstehung von gesundheitsrelevanten Handlungsspielräumen genauer erklären zu können. Die abschließende Diskussion liefert eine kurze Zusammenfassung, nennt spezifische Begrenztheiten und Potentiale des CA in seiner Anwendung für die Gesundheitsförderung und skizziert erste Ansatzpunkte für zukünftige Forschungen.

\section{Gesundheitsrelevante Handlungsspielräume als Gegenstand der Gesundheitsförderung}

Eine aktivierende und unterstützende Gesundheitsförderung, wie sie erstmals in der Ottawa-Charta (1986) formuliert wurde, betont die Bedeutung von gesundheitsförderlichen Lebensstilen. In ihren praktischen Bemühungen zielt die Gesundheitsförderung dementsprechend darauf ab, alle Menschen zu befähigen, gesundheitsförderliche Entscheidungen zu treffen und in konkrete Handlungen umzusetzen. Dieser Ausrichtung fehlt jedoch bis heute eine theoretische Begründung. Hier soll nun geprüft werden, inwieweit sich Amartya Sens Capability Approach (Sen 1999) als theoretische Fundierung für die Ungleichheitsforschung in einer so ausgerichteten Gesundheitsförderung eignet.

Beim Capability Approach (CA) handelt es sich um ein Paradigma, das ursprünglich aus der Entwicklungsökonomie stammt und sich nicht zuletzt deshalb als anwendungsorientiertes, aber dennoch theoretisch durchdachtes Konzept präsentiert. Zunächst sei ein grundlegendes Merkmal erwähnt, mit dem der CA über die Ressourcenansätze in der Tradition von Rawls' Theorie der Gerechtigkeit (Rawls 1971; Sen 1999; Ruger 2004) hinausgeht. Die Ressourcenansätze gehen davon aus, dass die Ungleichheit in der Verteilung von Ressourcen einen unmittelbaren Einfluss auf das gesundheitliche Wohlergehen hat. Gesundheit sei folglich ein Nebeneffekt sozialer Gerechtigkeit (Daniels, Kennedy und Kawachi 2000a), und Gesundheitsförderung müsse zuallererst bei der Verringerung sozialer Ungleichheit ansetzen (Daniels, Kennedy und Kawachi 2000b). In ihren gesellschaftspolitischen Implikationen sind die Ressourcentheorien hilfreich, indem sie in der Reduktion sozialer Ungleichheit eine notwendige Bedingung für die Milderung gesundheitlicher Ungleichheit erkennen. Wir gehen jedoch davon aus, dass zumindest in reichen Wohlfahrtsstaaten kein unmittelbarer und einheitlicher Kausalzusammenhang zwischen der Verfügbarkeit von Ressourcen, gesundheitlichem Verhalten und Wohlergehen besteht. D. h., eine Reduktion sozialer Ungleichheit ist zwar eine notwendige, aber nicht hinreichende Bedingung für Chancengleichheit in der Gesundheit. Entsprechend merkt Ruger (2004: 1093) an, dass eine Reduktion sozialer Ungleichheit nicht auch zwangsläufig zu einer Reduktion der Ungleichheit in der Gesundheit führt.

Im Kontrast zu den Ressourcentheorien rückt der CA nicht Ressourcen, sondern die persönlichen Verwirklichungschancen von Lebensentwürfen - und mit diesen eng ver- 
knüpft Gesundheit und Wohlergehen ${ }^{2}$ - in das Zentrum seiner Perspektive. So macht er Chancengleichheit nicht allein an der Verfügbarkeit materieller Ressourcen fest, sondern überdies an der Möglichkeit per se, ,bestimmte Lebensentwürfe verwirklichen zu können" (Arndt und Volkert 2006: 9). D. h., der CA befasst sich mit Handlungsoptionen, die auch, aber nicht ausschließlich an die Verfügbarkeit materieller Ressourcen gebunden sind (Marmot 2000). Wir gehen diesbezüglich nun davon aus, dass auch spezifisch gesundheitsrelevante Handlungsoptionen nur dann entstehen können, wenn dazu ein hinreichender Grundstock an materiellen Ressourcen besteht. Es ist jedoch denkbar, dass die Verfügbarkeit von materiellen Ressourcen ab einer gewissen Schwelle nur noch einen geringen Einfluss auf gesundheitsrelevante Handlungsoptionen hat (Marmot und Bell 2006: 29). So mag ein Mehr an materiellen Ressourcen zusätzlich zu einer bestehenden Ausstattung nur noch wenig Auswirkungen auf die Entscheidungen für gesundes Verhalten haben, andere Einflüsse dagegen (wie z. B. Werthaltungen und gesellschaftliche Normen) an relativer Bedeutung gewinnen.

Auf den Bereich der Gesundheitsförderung bezogen hieße dies, dass das Prinzip der Chancengleichheit nicht lediglich als formaler Anspruch auf eine hinreichende Ausstattung an Ressourcen für alle gelten soll, sondern dass im Idealfall niemand darin benachteiligt sein sollte, sich für einen gesundheitsförderlichen Lebensstil zu entscheiden und so den bestmöglichen Gewinn an Wohlergehen tatsächlich zu erreichen (Sen 2006: 23; Whitehead und Dahlgren 2006: 4 f.; Ridde, Guichard und Houeto 2007: 13). Dies bedeutet zum Beispiel, dass der Zugang zu gesundheitsförderlichen Bildungsangeboten oder medizinischen Leistungen nicht allein gesetzlich geregelt sein muss, sondern dass überdies geprüft werden muss, ob und mit welchem Aufwand diese Angebote genutzt werden können, d. h., inwiefern die Handlungsoptionen tatsächlich vorhanden sind. So kann im Rahmen des CA beispielsweise das Bewegungsverhalten in Alltagssituationen analysiert werden. Damit gemeint sind hier Aktivitäten wie etwa Treppensteigen oder zu Fuß gehen, die lediglich ein Minimum an materiellen Ressourcen und persönlichen Voraussetzungen erfordern. Trotzdem können Handlungsoptionen in Bezug auf das altägliche Bewegungsverhalten eingeschränkt șein, beispieisweise für Schülerinnen und Schüler, die einen risikoreichen Schulweg haben und deshalb von ihren Eltern zur Schule gefahren werden (Abel und Walter 2002; Shiell 2007), oder für Personen, die sog. Angsträume wie z. B. unbelebte öffentliche Plätze und dunkle Unterführungen meiden müssen.

\section{Capabilities and Achievements - Verwirklichungschancen und deren Realisierung in der Gesundheitsförderung}

Sen $(1993 ; 1999)$ unterstreicht den Unterschied zwischen Handlungsoptionen und tatsächlich realisierten Handlungen. Demnach umfasst der Raum der Verwirklichungschancen - der Capabilities - sämtliche tatsächlich möglichen Handlungen und Zustände, für die sich eine Person mit guten Gründen entscheiden kann. Dazu gehören nach Sen (Sen 1993: 36 f.) u. a. ,escaping morbidity and mortality, ... having mobility, ... being happy, ... taking part in the life of the community, ... being socially integrated." Während der Begriff der Capabilities auf den Raum der Verwirklichungschancen als Menge von idealiter tatsächlich möglichen Handlungen und Zuständen verweist, bezieht 
sich der Begriff der Functionings auf all jene Handlungen und Zustände, die realiter auch tatsächlich ins Werk gesetzt werden. „The capability of a person reflects the alternative combinations of functionings the person can achieve, and from which he or she can choose one collection" (Sen 1993: 31, Hervorhebung i. O.) Capabilities sind also realisierbare Potentiale (deshalb: Verwirklichungschancen), Functionings hingegen sind die davon tatsächlich realisierten Handlungen und Zustände: „A functioning is an achievement, whereas a capability is the ability to achieve" (Sen 1988: 36).

Wir verwenden im Folgenden den Begriff der „Capabilities", um auf den Bereich der potentiell möglichen Handlungen und Zustände zu verweisen, d. h., auf die Verwirklichungschancen und Handlungsspielräume, die eine Person hat. Der Terminus der „Functionings" bezieht sich dagegen auf tatsächlich vorhandene Zustände und Handlungen. Er fungiert als Sammelbegriff für Handlungen und Zustände, die aus guten Gründen vollzogen bzw. erreicht werden, seien sie nun aktiv angestrebt worden oder nicht. Tatsächliche Zustände können theoretisch auch ohne das aktive Zutun von Individuen eintreten (eine schicksalhafte Erkrankung, Zugangsverlust durch Schließung einer Bildungsstätte usw.). Um eine entsprechende Differenzierung zu ermöglichen, beziehen wir (mit Sen) den Begriff „Achievement" speziell auf all jene Handlungen und Zustände, die durch aktives, zielgerichtetes und wertebasiertes Streben erreicht werden (vgl. Abb. 1). In unserem Blickwinkel der verhaltensbezogenen Gesundheitsförderung wird im Folgenden der Fokus auf Achievements gelegt, und damit auf die Teile des persönlichen Wohlergehens, die auf dem aktiven Zutun von Personen und Kollektiven beruhen. Um beim Beispiel des alltäglichen Bewegungsverhaltens zu bleiben: Mobilität ist ein Functioning, für das sich eine Person mit guten Gründen entscheiden kann; Capabilities hingegen sind die Handlungsoptionen, etwa für körperliche Aktivitäten auf Schul- oder Arbeitswegen; die konkreten Situationen schließlich, in denen sich Personen zu Fuß oder mit dem Fahrrad fortbewegen, sind Achievements.

Mit Sen gehen wir davon aus, dass „Basic Capabilities“ (Sen 1985: 46) - wie z. B. ausreichende Ernährung oder die Vermeidung von Krankheiten - im Kontext von reichen Wohlfahrtsstaaten für den allergrößten Teil der Bevölkerung realisierbar sind (s. a. Marmot und Bell 2006: 29). Dies ermöglicht eine Fokussierung auf die durch relativ große Wahlfreiheit charakterisierten Elemente der westlichen Lebensstile und insbesondere deren Relevanz für die Gesundheit und das persönliche Wohlergehen (Robeyns 2005: 101; Sen 2006: 23). Über die gesundheitliche Bedeutung spezifischer Lebensstile wird so der CA auch für eine theoretisch-konzeptionelle Fundierung der Gesundheitsförderung in westlichen wohlhabenden Gesellschaften anwendbar.

Gesundheitliches Wohlergehen kann im CA als ein Functioning verstanden werden, welches zu großen Teilen durch die Umsetzung von Handlungsoptionen (Capabilities) in tatsächliches Gesundheitsverhalten (Achievement) erreicht wird. Aus einer soziologischen Sicht ist davon auszugehen, dass sowohl der Raum der Capabilities als auch die erfolgreiche Umsetzung der Optionen durch die Verfügbarkeit von Handlungsressourcen und durch spezifische Kontextbedingungen beeinflusst werden. Mit anderen Worten, der Raum der gesundheitsrelevanten Capabilities und damit der gesundheitsförderlichen Handlungsoptionen wird durch die jeweils vorhandenen Ressourcen und Kontexte aufgespannt. Die Ressource „Fahrrad“ beispielsweise schafft die Möglichkeit, den Schul- oder Arbeitsweg durch eigene körperliche Bewegung zu bewältigen. Dazu müs- 
sen jedoch gewisse Randbedingungen erfüllt sein: Das Klima muss sich dafür eignen, es müssen sichere Verkehrswege vorhanden sein, an den Schulen und Arbeitsplätzen muss eine geeignete Infrastruktur zur Verfügung stehen ( $z$. B. sichere und gedeckte Abstellplätze, Duschen), etc.

Die Unterscheidung von Capabilities und Achievements wird von Sen deshalb so stark betont, weil er die Ansicht vertritt, dass das Wohlergehen einer Person nicht einzig aufgrund der Achievements selber bewertet werden soll, sondern dass die Möglichkeit, zwischen alternativen Handlungsoptionen zu wählen, eine Freiheit sui generis ist (Sen 1999: 74) - etwa die Möglichkeit, sich dafür zu entscheiden, den Arbeitsweg mit dem Fahrrad zurückzulegen, auch wenn diese Möglichkeit nicht täglich wahrgenommen wird. Deshalb sollte als Bewertungsrahmen von entwicklungs- und sozialpolitischen Interventionen - und, so möchten wir hier anfügen: gesundheitspolitischen Interventionen - der Raum der Capabilities herangezogen werden und nicht ausschließlich die Qualität oder Quantität der verfügbaren Ressourcen oder die realisierten Handlungen und Zustände. Tatsächlich fokussiert der CA auf eine Analyseebene, die den Nexus zwischen Ressourcen und Achievements bildet (vgl. Abb. 1).

\section{Abb. 1: Der Capability Approach als Erklärungsmodell in der Gesundheitsförderung}

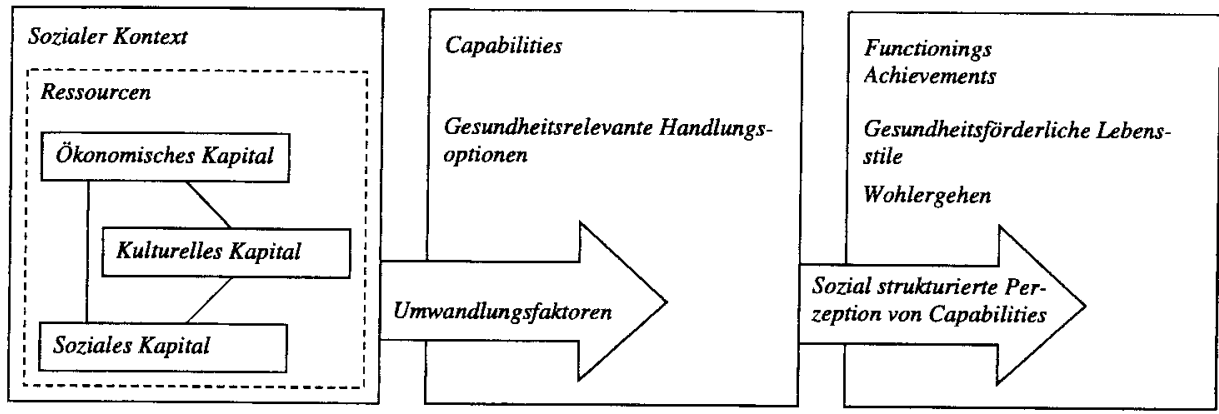

\section{Gesundheitsrelevante Handlungsspielräume: Ressourcen und Umwandlungsfaktoren}

"We use incomes and commodities as the material basis of our well-being. But what use we can respectively make of a given bundle of commodities, or more generally of a given level of income, depends crucially on a number of contingent circumstances, both personal and social" (Sen 1999: 70).

Der CA geht davon aus, dass die Beziehung zwischen Ressourcen (Sen spricht von Commodities) und dem, was man mit ihnen effektiv tun kann, um das persönliche Wohlergehen sicher zu stellen (den Achievements), von persönlichen und kontextuellen Umwandlungsfaktoren strukturiert ist (Sen 1985:25 f.). Der Begriff der Umwandlungsfaktoren (Arndt und Volkert 2006: 10) zielt darauf ab, die Vielfalt menschlicher Lebensweisen zu unterstreichen und die von Ressourcentheorien postulierte unilineare Beziehung zwischen Einkommen und Wohlergehen grundsätzlich in Frage zu stellen (Sen 1988: 16, 1993: 41): „The impact of income on capabilities is contingent and conditional“ ( $\operatorname{Sen} 1999: 88)$. 
Den Einfluss persönlicher Faktoren, welche die Transformation von Einkommen in Wohlergehen beeinflussen, zeigt Sen $(1985 ; 1999$ : 69) am Beispiel der Ernährung auf. Der Zusammenhang zwischen Nahrungsmitteln (Ressource) und der Aufnahme von Kalorien hängt von einer Vielzahl physiologischer Faktoren ab: Um eine angemessene Ernährung und damit Wohlergehen zu erzielen, benötigen verschiedene Personen unterschiedliche Mengen an Nahrung. So benötigen etwa schwangere oder stillende Frauen oder auch Personen, die eine körperlich anstrengende Arbeit verrichten, mehr Nahrungsmittel. Andere Autoren führen darüber hinaus erworbene persönliche Eigenschaften wie Lesekompetenz oder Intelligenz als weitere persönliche Umwandlungsfaktoren an (z. B. Robeyns 2005: 99).

Sen nennt drei Bereiche, in denen kontextuelle Faktoren eine Rolle spielen, die sich vom Individuum nur bedingt kontrollieren lassen: (1) Der räumliche Kontext wirkt auf die Umwandlungsfaktoren z. B. aufgrund der Exposition von Naturgefahren oder von Gewalt in urbanen Räumen ein. (2) Der epidemiologische Kontext hat Einfluss auf die Umwandlungsfaktoren z. B. durch Krankheiten, die in einer Gegend endemisch sind. (3) Der soziale Kontext schließlich verweist u. a. auf Normen und Werte (z. B. solche zur Rollenverteilung zwischen den Geschlechtern oder spezifischen Verpflichtungen gegenüber der Familie), welche die Umwandlungsfaktoren zwischen Ressourcen und Capabilities beeinflussen (Sen 1999: 88). Unterschiedliche Umwelten - räumliche, epidemiologische und soziale - haben also eine unmittelbare Auswirkung auf das Ausmaß, inwieweit die verfügbaren Ressourcen nützliche Handlungsoptionen eröffnen.

Im vorherigen Beispiel der Gesundheitsförderung: Der Besitz eines Fahrrads als Ressource für Bewegung im Alltag eröffnet nur dann gesundheitsrelevante Handlungsspielräume, wenn zum einen die persönlichen Voraussetzungen erfüllt sind und zum anderen die Umwandlungsfaktoren im Vergleich zu anderen Ressourcen wie z. B. dem öffentlichen Nahverkehr oder einem Privatwagen ungefähr in gleichem Maße gegeben sind. $\mathrm{Zu}$ den Bedingungen, welche die Umwandlungsfaktoren negativ beeinflussen können, gehören z. B. große Distanzen zwischen Wohn- und Arbeitsort, ungünstige Klimabedingungen, unsichere Verkehrswege (physische Umwelt), aber auch gesellschaftliche Erwartungshaltungen in Bezug auf eine dem sozialen Status angemessene Wahl des Verkehrsmittels (sozialer Kontext).

Sen streicht insbesondere mit dem Begriff der kontextuellen Umwandlungsfaktoren heraus, dass Achievements nicht ohne Berücksichtigung des sozialen Umfeldes der handelnden Personen verstanden werden können. Für die Gesundheitsförderung eröffnen sich damit Anschlussmöglichkeiten an ihr Konzept der Settings, das explizit davon ausgeht, dass gesundheitsförderliche Interventionen in soziale und institutionelle Kontexte eingebunden werden sollen (Whitelaw et al. 2001).

\section{Gesundheitsrelevante Ressourcen: Ökonomisches, kulturelles und soziales Kapital}

Die Adaption des CA für die Gesundheitsförderung macht es erforderlich, dass die Ressourcen genauer differenziert werden, denn es sind nicht allein materielle Ressourcen wie Einkommen oder Naturalien, die gesundheitsrelevante Handlungsoptionen eröff- 
nen. Auf der Suche nach den Einflussfaktoren auf gesundheitsrelevante Handlungsspielräume möchten wir dazu ökonomische, kulturelle und soziale Ressourcen unterscheiden, welche in Form von Kapitalien miteinander interagieren und die (gesundheitsförderlichen) Handlungschancen bestimmen (vgl. hierzu auch Abel, Abraham und Sommerhalder 2006; Abel 2007). Mit Bourdieu (1983) gehen wir davon aus, dass Kapital „akkumulierte Arbeit [ist], entweder in Form von Materie oder in verinnerlichter, ,inkorporierter ' Form.“ Es ist zugleich ,eine Kraft, die den objektiven und subjektiven Strukturen innewohnt [und] grundlegendes Prinzip der inneren Regelmäßigkeiten der sozialen Welt" (Bourdieu 1983: 183). Ökonomisches Kapital zeigt sich als finanzielle Ressourcen und Eigentum. Insbesondere in Form von Geld erweitert es direkt die Handlungsspielräume auch für gesundheitsrelevantes Verhalten. Kulturelles Kapital manifestiert sich in drei verschiedenen Ausprägungen (Bourdieu 1983): Erstens als objektiviertes kulturelles Kapital in Form von Gegenständen wie Büchern, Werkzeugen oder Geräten, zweitens als institutionalisiertes kulturelles Kapital in Form von Bildungstiteln und Berufszertifikaten und drittens schließlich als inkorporiertes - d. h. qua Sozialisation verinnerlichtes - kulturelles Kapital in Form von Normen, Werten, Wissen und praktischen Fertigkeiten (Veenstra 2007; Abel 2008). Insbesondere in seiner inkorporierten Form prägt und strukturiert kulturelles Kapital die Handlungsmöglichkeiten und -spielräume der Menschen. Unter dem Begriff des sozialen Kapitals ${ }^{3}$ versteht Bourdieu (1983) sowohl ein Potential von Ressourcen wie auch die Ressourcen selber, die über interpersonelle Beziehungen mobilisiert werden können: „Das Sozialkapital ist die Gesamtheit der aktuellen und potentiellen Ressourcen, die mit dem Besitz eines dauerhaften Netzes von mehr oder weniger institutionalisierten Beziehungen gegenseitigen Kennens oder Anerkennens verbunden sind" (Bourdieu 1983: 190). Das Zusammenspiel von ökonomischem, sozialem und kulturellem Kapital, so eine am anderen Ort entwickelte These (Abel, Abraham und Sommerhalder 2006; Abel 2007), bestimmt die Gesundheitschancen der Menschen speziell über die Strukturierung und Erweiterung gesundheitsförderlicher Handlungsspielräume.

\section{Perzeption und Nutzungsbedingungen von gesundheitsrelevanten Handlungsoptionen}

Das Zusammenspiel von Ressourcen und Umwandlungsfaktoren eröffnet einen Raum der Handlungsoptionen, innerhalb dessen sich alle theoretisch möglichen Functionings realisieren lassen (vgl. Abb. 1). Die Entscheidung, welche Functionings schließlich realisiert werden, liegt vorwiegend bei den Akteuren; sie wird nach Sen einzig durch gesellschaftlich geteilte, allgemeine normative Werte eingeschränkt.

Sen begnügt sich hier mit einer Konzeption der individuellen Auswahl von Functionings, die zwar nicht gänzlich vom sozialen Kontext gelöst ist, den Akteuren jedoch eine relativ große Wahlfreiheit einräumt. ${ }^{4}$ Er gehe davon aus, so Robeyns (2005: 109), dass sich Menschen rational verhielten und sich sozialem und moralischem Druck, der von Gruppen ausgehe, auch erfolgreich widersetzen könnten. Aus einer soziologischen Perspektive liegt hier nun ein wichtiger Schwachpunkt des CA, nämlich die Vernachlässigung des Sozialen. So macht Max Webers Begriff der Lebensführung deutlich, dass 
Handlungsoptionen nicht allein von „Gemeinsamen Normen“, „herrschende[n] Werte[n] und soziale[n] Sitten ${ }^{\text {"5 }}$ eingeschränkt werden, sondern dass sie darüber hinaus auch an die verfügbaren Ressourcen zurückgebunden sind (s. u. a. auch Cockerham, Abel und Lüschen 1993).

Stewart hat denn auch eine Öffnung des CA gegenüber Theorien des Sozialen vorgeschlagen, damit der Einfluss von sozialen Gruppierungen wie Familien oder ethnischen Gemeinschaften auf die Präferenzen in der Wahl der Capabilities berücksichtigt werden kann: , , . . . S Society - and indeed particular groups within society - shapes every individual, influencing preferences and consequent choices" (Stewart 2005: 189, Hervorhebung i. O.)

Tatsächlich könnte man mit den Bourdieu'schen Begriffen des Habitus und des kulturellen Kapitals (Bourdieu 1979; 1983) einwenden, dass die objektivistische Konzeption der Capabilities bei Sen verkennt, dass schon die Perzeption der Wahlmöglichkeiten sozial strukturiert ist. D. h., die Wahrnehmung von und die Auswahl aus objektiv realisierbaren Capabilities wird eingeschränkt, wenn sie von Personen subjektiv als nicht realisierbar oder als höchst unwahrscheinlich wahrgenommen werden. Das wirft die Frage auf, ob theoretisch und von außen betrachtet realisierbare Lebensentwürfe auch tatsächlich verwirklicht werden können oder ob sie von einem lagespezifischen Habitus, also einer „Handlungs-, Wahrnehmungs- und Denkmatrix (Bourdieu 1979: 168)“ verwischt werden, d. h. vom Individuum nicht perzipiert und folglich auch nicht umgesetzt werden können. Bourdieu geht nämlich davon aus, dass der Habitus an die „materiellen Existenzbedingungen“ (1979: 164) zurückgebunden ist, also an die Verfügbarkeit von ökonomischem, kulturellem und sozialem Kapital. Das inkorporierte kulturelle Kapital spielt dabei eine tragende Rolle, da es ein „Besitztum [ist], das zu einem festen Bestandteil der ,Person“, zum Habitus geworden ist“" (Bourdieu 1983: 187).

Es gilt deshalb zu prüfen, inwiefern die Perzeption und die Nutzungsbedingungen von gesundheitsrelevanten Handlungsspielräumen mit den verfügbaren ökonomischen, sozialen und kulturellen Ressourcen im Zusammenhang stehen. Dies könnte einzig durch eine empirische Analyse geschehen, welche Ressourcen, Capabilities und Achievements differenziert. Ausgehend von den Ressourcen müsste sie in einem ersten Schritt den Raum gesundheitsrelevanter Handlungsspielräume abstecken. Aus dem Passungsverhältnis zwischen Handlungsspielräumen und Achievements ließen sich in einem zweiten Schritt Indizien zu den Nutzungsbedingungen der Capabilities gewinnen, die möglicherweise sozial differenziell verteilt sind. Werden schließlich die Nutzungsbedingungen zu den Ressourcen ins Verhältnis gesetzt, ließe sich beurteilen, inwiefern die Perzeption von gesundheitsrelevanten Capabilities an einen lagespezifischen Habitus gebunden ist. Würden die verfügbaren ökonomischen, sozialen und kulturellen Ressourcen stark mit der Perzeption der Handlungsspielräume korrelieren, so spräche dies für eine Sozialisationstheorie Bourdieu'schen Zuschnitts. Wäre die Assoziation der erwähnten Merkmale jedoch nur schwach, so ließe sich dies als Hinweis darauf deuten, dass Personen ihre Handlungsoptionen relativ frei und unabhängig von ihrer sozialen Position umsetzen könnten.

Solche Überlegungen zeigen mögliche Schnittstellen des CA zur empirischen Ungleichheitsforschung in der Gesundheitsförderung auf. Sie verdeutlichen zudem den Kern des CA, wenn es um dessen Anwendung im Hinblick auf gesundheitsförderliche 
Interventionen geht. Es stellt sich nämlich die Frage, welche Einflüsse Interventionen, die eine Ausweitung der gesundheitsrelevanten Handlungsoptionen zum Ziel haben, tatsächlich auf das gesundheitsrelevante Verhalten, d. h. auf die Achievements, haben.

\section{Die Chancen und Grenzen des CA in der Gesundheitsförderung}

Armatya Sen geht es in seinen Arbeiten um die Ausweitung der Capabilities an sich: die Menschen sollten nach ihren selbstbestimmten Bedürfnissen handeln können. Eine Ausweitung der Handlungsoptionen ist eine Errungenschaft schon deshalb, weil jeder Zuwachs an Selbstbestimmung - um mit Sen (1999) zu sprechen - an sich schon ein Schritt in Richtung Freiheit bedeutet. Dabei geht er davon aus, dass Menschen in der Regel wissen, was das Richtige für sie ist (Sen 1993; 1999). Aus der Sicht der Gesundheitsförderung ist dabei zu beachten, dass mit der Erweiterung von Handlungsspielräumen grundsätzlich sowohl die Möglichkeiten für gesundheitsförderliches als auch für gesundheitsschädigendes Handeln vergrößert werden können (z. B. die Entscheidung zu Rauchen).

Eine Gesundheitsförderung, die über die Ausweitung von Handlungsspielräumen wirken möchte, muss zu diesem potentiellen Konflikt zwischen Handlungsfreiheit per se (einschließlich Risikoverhalten) und gesundheitsförderlicher Handlungsfreiheit Stellung beziehen. Dazu können hier nur einige wenige Gedanken als Startpunkt entsprechender Überlegungen genannt werden. So gilt es zu beachten, dass es im menschlichen Handeln letztlich immer individuelle Entscheidungen geben wird (und in einer liberalen Gesellschaftsordnung immer geben soll), die die Gesundheit beeinträchtigen oder gar schädigen können. Während solche Präferenzen des Einzelnen akzeptiert werden müssen, kann sich jedoch die Gesundheitsförderung bei der Verbesserung der Handlungsspielräume auf jene Capabilities konzentrieren, die ein hohes Potential an Gesundheitsverbesserung in sich tragen; ein Beispiel hierfür wären die Zugangschancen zu gesundheitsförderlichen Bewegungs- und Begegnungsräumen. Mit der vermehrten Schaffung solcher Räume (z. B. spielfreundliche Parks) würden gezielt Handlungsoptionen für gesundheitsförderliches Verhalten erweitert und damit wichtige Ziele der Gesundheitsförderung erreicht: verbesserte Bedingungen des Gesundheitsverhaltens, Förderung der aktiven individuellen Entscheidung für Gesundheit sowie die Frweiterung von Handlungsspielräumen im Sinne des Empowerment. Outcome-orientierte Evaluationen entsprechender Interventionen können in diesem Sinne zwischen den verbesserten Handlungsoptionen (Capabilities), der tatsächlichen Nutzung solcher Handlungs(spiel)räume und der gegebenenfalls erzielten Verbesserung des Wohlbefindens (Achievements) als Zicle auf unterschiedlichen Ergebnisebenen differenzieren (Ruckstuhl und Abel 2001; Spencer et al. 2008). Um beim oben genannten Beispiel zu bleiben: Ein breiteres Angebot an Bewegungs- und Begegnungsräumen vergrößert die Handlungsoptionen, erhöht die Wahrscheinlichkeit von mehr körperlicher Bewegung der Menschen, erhöht ihre Chancen für mehr soziale Kontakte, reduziert die Wahrscheinlichkeit von sozialer Isolation und kann so in verschiedener Weise zu mehr Lebensfreude beitragen. In dieser Form wären mittels des Capability-Ansatzes gleich mehrere wichtige Ziele der Gesundheitsförderung erreicht. 


\section{Zusammenfassung: Handlungsspielräume als Zielgröße der Gesundheitsförderung}

Laut ihrem Paradigma zielt die moderne Gesundheitsförderung auf die Verbesserung der Gesundheit und dabei insbesondere auf die Verbesserung der Gesundheitschancen in bisher benachteiligten Bevölkerungsgruppen (Concept and Principles 1986). Sowohl bei der Schaffung von gesunden Lebensbedingungen wie auch bei der Förderung von gesundheitszuträglichen Verhaltensmustern baut sie auf Empowerment und die aktive Beteiligung der Bevölkerung. Für die Verbindung ihrer Interventionsansätze mit den immanenten Fragen der sozialen Ungleichheit fehlen der Gesundheitsförderung allerdings bis heute die passenden Forschungsmodelle und dementsprechend die empirisch belegten Wissensbestände: Welche Zusammenhänge bestehen zwischen sozialer Lage, Handlungsspielräumen und Gesundheitsstatus, und wie könnten Maßnahmen zum Empowerment hier wirksam sein? Wie lassen sich mittels Partizipation die Optionen für gesundheitsförderliches Handeln von Menschen in sozial schwierigen Lagen verbessern und inwiefern trägt das zur Steigerung ihrer gesundheitsbezogenen Lebensqualität bei? Wenn in der Gesundheitsförderung das Erkenntnisinteresse primär auf Gesundheitsentwicklung, auf soziale Lebensbedingungen, auf die Ressourcenverteilung und auf lagespezifische Verhaltensmuster gelegt wird, dann muss auch die Forschung entsprechend ausgerichtet werden. Marmot und Bell (2006) weisen in einer neueren Arbeit daraufhin, dass Ungleichheit in gesundheitlichen Belangen auch auf die Ungleichheit in den Handlungsoptionen zurückgeht: „Social inequalities in health may be a consequence of inequalities in capabilities"(2006: 27). Dies legt nahe, dass sich die Gesundheitsförderung auf eine gezielte Erweiterung gesundheitsförderlicher Capabilities in solchen Bevölkerungsgruppen konzentrieren sollte, welche deutlich eingeschränkte Handlungsoptionen aufweisen.

Wir sehen in Amartya Sens Capability Approach (CA) ein vielversprechendes Paradigma, das den Forschungsgegenständen der Gesundheitsförderung in umfassender Weise gerecht wird. Erstens operiert der CA mit dem zentralen Begriff des Wohlergehens, der sich als positiv definiertes Gesundheitsmaß sowohl auf Personen und Kollektive beziehen kann. Zweitens eröffnen sich mit dem Begriff der kontextuellen Umwandlungsfaktoren Anschlussmöglichkeiten an das Konzept der Settings, das explizit davon ausgeht, dass gesundheitsförderliche Interventionen in sozialen und institutionellen Kontexten eingebunden werden sollen. Drittens nimmt er eine akteurzentrierte Perspektive ein, indem er davon ausgeht, dass Individuen und Kollektive ihre soziale und physische Umwelt aktiv gestalten. Und viertens schließlich richtet der CA das Augenmerk auf jene Prozesse, die an den Schnittstellen zwischen (materiellen und immateriellen) Ressourcen, Handlungsoptionen und den tatsächlichen Handlungen liegen. Diese analytische Differenzierung erlaubt es, die Perzeption und die Nutzungsbedingungen von Handlungsspielräumen zu erforschen. Allerdings muss die Forschung zukünftig besondere Anstrengungen unternehmen, um die Frage zu beantworten, ob und inwieweit die Umsetzung gesundheitsförderlicher Handlungsoptionen von einem lagespezifischen Habitus bestimmt wird, oder ob alle Personen ungeachtet ihrer sozialen Position gegebene Handlungsspielräume gleichermaßen perzipieren und nutzen - also im doppelten Sinne des Wortes wahrnehmen. Dazu, so haben wir versucht zu zeigen, bedarf der CA gezielter Ergänzungen. Wir haben entsprechend vorgeschlagen, die ungleiche Vertei- 
lung von Nutzungsbedingungen und Perzeptionsmustern über die Bourdieu'schen Begriffe der Kapitalien und des Habitus zu erklären.

Der Capability Approach in der Gesundheitsförderung zeigt sich somit insgesamt als eine sinnvolle Ergänzung der heute etablierten Forschungs- und Interventionsansätze zur gesundheitlichen Ungleichheit. Die Gesundheitsförderung könnte in der Betonung salutogenetischer Prozesse ihre Schwerpunkte auf die aktive Rolle der Menschen in ihrer Lebensgestaltung und in ihren Beiträgen zur Gemeinschaft legen. Sie hat mit dem Capability Approach dazu eine tragfähige theoretische Grundlage.

\section{Anmerkungen}

1 Für eine detailliertere Auseinandersetzung mit dem CA siehe u. a. Sen (1993; 1999) Robeyns (2005), Comim, Qizilbash und Alkire (2008).

2 Wir verstehen hier Gesundheit als eine Form des ,,well-being“. Dieser Begriff wurde in der Ottawa-Charta verwendet mit dem Ziel, das Gesundheitsverständnis über im engeren Sinne medizinisch behandlungsbedürftige Zustände hinaus zu erweitern. In den Schriften Sens (1993; 1999) findet der Begriff, ,well-being" Verwendung, um das personengebundene (auch auf Kollektive inkl. ganzer Bevölkerungen beziehbare) Ziel entwicklungspolitischer Maßnahmen zu definieren. Wir übertragen den englischsprachigen Begriff des ,well-being“ in seiner Verbindung mit Gesundheit als „Wohlergehen“ oder „Wohlbefinden“ ins Deutsche. Erstens repräsentieren beide Begriffe Gesundheit als positiv definierten Zustand oder Prozess (d. h. nicht primär als Abwesenheit von Krankheit). Zweitens enthalten die Begriffe Wohlbefinden und Wohlergehen ein starkes subjektives Element, das die Bedeutung kultureller Wertmaßstäbe im Gegensatz zu medizinisch-objektivierten Bewertungskriterien - unterstreicht. Da der Begriff des Wohlbefindens eher auf einen individuellen, häufig flüchtigen Status verweist, verwenden wir im Folgenden meist den Begriff des Wohlergehens. Mit der Verwendung des Begriffs „Wohlergehen" möchten wir die Prozesshaftigkeit von Gesundheit unterstreichen. Letztlich lässt sich der Begriff des Wohlergehens auch auf Kollektive anwenden (z. B. das Wohlergehen einer sozialen Gruppe.)

3 Für die Diskussion zum Begriff des sozialen Kapitals in der Sozialepidemiologie vgl. Hawe und Shiell (2000), Macinko und Starfield (2001), Muntaner und Lynch (2002), Moore et al. (2006).

4 Sen interessiert sich weniger dafür, auf welche Weise die sozialen Kräfte die Perzeption und die Nutzungsbedingungen von Handlungsspielräumen und folglich ebenfalls die Handlungen in die eine oder andere Richtung lenken. Vielmehr geht es ihm darum zu zeigen, dass der Raum der Capabilities eine relevante Analyseebene darstellt. Anders als wir es hier tun, stützt er sich auf den Begriff der Auswahl, wenn er die Verknüpfung zwischen dem Raum der Capabilities und den tatsächlichen Handlungen (Achievements) herstellt: „The capability of a person reflects the alternative combinations of functionings the person can achieve, and from which he or she can choose one collection" (1993: 31, Hervorhebung i. O.).

5 Vgl. Sen (2000: 20). Im Original: „Social values and prevailing mores", ,,shared norms“, „prevailing values and social mores" (1999: 9).

\section{Literatur}

Abel, Thomas. 1991. Measuring Health Lifestyles in a Comparative Analysis. Theoretical Issues and Empirical Findings. Social Science and Medicine 32 (8): 899-908.

Abel, Thomas. 2007. Cultural Capital in Health Promotion. In: Health and Modernity. The Role of Theory in Health Promotion, hrsg. David C. McQueen und Ilona Kickbusch, 43-73. Berlin: Springer. 
Abel, Thomas. 2008. Cultural Capital and Social Inequality in Health. Journal of Epidemiology \& Community Health 62 (8).

Abel, Thomas, Andrea Abraham und Kathrin Sommerhalder. 2006. Kulturelles Kapital, kollektive Lebensstile und die soziale Reproduktion gesundheitlicher Ungleichheit. In: Gesundheitliche Ungleichheit: Grundlagen, Probleme, Perspektiven, hrsg. Matthias Richter und Klaus Hurrelmann, 185-198. Wiesbaden: Verlag für Sozialwissenschaften.

Abel, Thomas, William C. Cockerham und Steffen Niemann. 2000. A Critical Approach to Lifestyle and Health. In: Researching Health Promotion, hrsg. Jonathan Watson und Stephen Platt, 54-77. London: Routlege.

Abel, Thomas und Esther Walter. 2002. Individuelles Verhalten und soziale Verantwortung für Gesundheit: Von der Wissenschaft zur Praxis der Gesundheitsförderung. In: Gesundheitsforrderung - Eine neue Zeitforderung. Interdisziplinäre Grundlagen und Beitrag der Komplementärmedizin, hrsg. Peter Heusser, 31-42. Bern: Peter Lang.

Arndt, Christian und Jürgen Volkert. 2006. Amartya Sens Capability-Approach - Ein neues Konzept der deutschen Armuts- und Reichtumsberichterstattung. Vierteljahrshefte zur Wirtschaftsforschung 75 (1): 7-29.

Beck, Ulrich. 1986. Risikogesellschaft: Auf dem Weg in eine andere Moderne. Frankfurt a. M.: Suhrkamp.

Bisig, Brigitte, Matthias Bopp und Christoph E. Minder. 2001. Sozio-ökonomische Ungleichheit und Gesundheit in der Schweiz. In: Sozialepidemiologie. Eine Einführung in die Grundlagen, Ergebnisse und Umsetzungsmöglichkeiten, hrsg. Andreas Mielk und Kim Bloomfield, 60-71. München: Juventa Verlag.

Blaxter, Mildred. 1990. Health and Lifestyles. London: Travistock.

Bourdieu, Pierre. 1979. Entwurf einer Theorie der Praxis auf der ethnologischen Grundlage der kabylischen Gesellschaft. Frankfurt a. M.: Suhrkamp.

Bourdieu, Pierre. 1983. Ökonomisches Kapital, kulturelles Kapital, soziales Kapital. In: Soziale Welt, Sonderheft 2: Soziale Ungleichheiten, hrsg. Reinhard Krekel: 183-198.

Cockerham, William C. 2005 . Health lifestyle theory and the convergence of agency and structure. Journal of Health and Social Behavior 46 (1): 51-67.

Cockerham, William C., Thomas Abel und Günther Lüschen. 1993. Max Weber, formal rationality and health lifestyles. Sociological Quarterly 34: 413-425.

Cockerham, William C., Alfred Rütten und Thomas Abel. 1997. Conceptualizing Contemporary Health Lifestyles: Moving Beyond Weber. Sociological Quarterly 38 (2): 321-342.

Comim, Flavio, Mozaffar Qizilbash und Sabina Alkire, hrsg. 2008. The Capability Approach. Concepts, Measures and Applications. Cambridge: Cambridge University Press.

Concept and Principles (A discussion document on the concept and principles of health promotion). 1986. Health Promotion International 1 (1): 73-76.

Currie, Candace, Saoirse Nic Gabhainn, Emmanuelle Godeau, et al., hrsg. 2008. Inequalities in Young People's Health. HBSC International Report from the 2005/2006 Survey. Copenhagen: WHO Regional Office for Europe.

Daniels, Norman, Bruce P. Kennedy und Ichiro Kawachi. 2000a. Is Inequality Bad for Our Health? Boston: Beacon Press.

Daniels, Norman, Bruce P. Kennedy und Ichiro Kawachi. 2000b. Justice is Good for Our Health. How greater economic equality would promote public health. Boston Review, http://bostonreview.net/BR25.1/daniels.html. 9. 1. 2009.

Droomers, M., C. T. M. Schrijvers und J. P. Mackenbach. 2001. Educational level and decreases in leisure time physical activity: predictors from the longitudinal GLOBE study. Journal of Epidemiology \& Community Health 55 (8): 562-568.

Frohlich, Katherine L., Ellen Corin und Louise Potvin. 2001. A theoretical proposal for the relationship between context and disease. Sociology of Health \& Illness 23 (6): 776-797. 
Giddens, Anthony. 1991. Modernity and Self-Identity. Self and Society in the Late Modern Age. Stanford: Stanford University Press.

Hawe, Penelope und Alan Shiell. 2000. Social Capital and Health Promotion: a Review. Social Science and Medicine 51: 871-885.

Helmert, Uwe, Karin Bammann und Rainer Müller, hrsg. 2000. Müssen Arme früher sterben? Soziale Ungleichheit und Gesundheit in Deutschland. Weinheim: Juventa.

Kouvonen, Anne, Mika Kivimaki, Ari Vaananen, et al. 2007. Job Strain and Adverse Health Behaviors: The Finnish Public Sector Study. Journal of Occupational \& Environmental Medicine 49 (1): 68-74.

Lampert, Thomas, Anke-Christine $\mathrm{Saß}$, Michael Häfelinger, et al. 2005. Armut, soziale Ungleichheit und Gesundheit: Expertise des Robert Koch-Instituts zum 2. Armuts- und Reichtumsbericht der Bundesregierung. Berlin: Robert Koch Institut.

Macinko, James und Barbara Starfield. 2001. The Utility of Social Capital in Research on Health Determinants. The Milbank Quarterly 79 (3): 387-427.

Mackenbach, Johan P. 2006. Health Inequalities: Europe in Profile. Rotterdam: University Medical Center Rotterdam. http://www.dh.gov.uk/prod_consum_dh/groups/dh_digitalassets/@dh/ @en/documents/digitalasset/dh_4121584.pdf. 9. 1. 2009.

Marmot, Michael. 2000. Do Inequalities Matter? Boston Review, http://bostonreview.net/BR25.1/ marmot.html. 9. 1. 2009.

Marmot, Michael. 2006. Health in an unequal world. The Lancet 368 (9552): 2081-2094.

Marmot, Michael und Ruth Bell. 2006. The Socioeconomically Disadvantaged. In: Social Injustice and Public Health, hrsg. Barry Levy und Victor Sidel, 25-45. Oxford: Oxford University Press.

McQueen, David C. und Laurie M. Anderson. 2001. What counts as evidence: issues and debates. In: Evaluation in health promotion. Principles and perspectives, hrsg. Irving Rootman, Michael Goodstadt, Brian Hyndman, et al., 63-81. Copenhagen: World Health Organization.

Mielck, Andreas. 2005. Soziale Ungleichheit und Gesundheit. Einführung in die aktuelle Diskussion. Bern: Hans Huber.

Moore, S., V. Haines, P. Hawe, et al. 2006. Lost in translation: a genealogy of the „social capital“ concept in public health. Journal of Epidemiology and Community Health 60 (8): 729-734.

Muntaner, Carles und John Lynch. 2002. Social capital, class, gender and race conflict, and population health: an essay review of Bowling Alone's implications for social epidemiology. International Journal of Epidemiology 31 (1): 261-267.

Ottawa-Charta zur Gesundheitsförderung. http://www.euro.who.int/AboutWHO/Policy/20010 827_2?language $=$ German. 9. 1. 2009.

Rawls, John. 1971. A Theory of Justice. Cambridge: Harward University Press.

Richter, Matthias. 2005. Gesundheit und Gesundheitsverhalten im Jugendalter. Der Einfluss sozialer Ungleichheit. Wiesbaden: Verlag für Sozialwissenschaften.

Richter, Matthias und Klaus Hurrelmann, hrsg. 2006. Gesundheitliche Ungleichheit. Grundlagen, Probleme, Perspektiven. Wiesbaden: VS Verlag für Sozialwissenschaften.

Ridde, Valery, Anne Guichard und David Houeto. 2007. Social inequalities in health from Ottawa to Vancouver: action for fair equality of opportunity. Promotion \& Education 14 (2_suppl): $12-16$.

Robeyns, Ingrid. 2005. The capability approach: a theoretical survey. Journal of Human Development 6 (1): 93-114.

Ruckstuhl, Brigitte und Thomas Abel. 2001. Ein Modell zur Typisierung von Ergebnissen der Gesundheitsförderung: eine kommentierte Darstellung des Ansatzes von Don Nutbeam. Prävention 24: 35-37.

Ruger, Jennifer Prah. 2004. Ethics of the social determinants of health. The Lancet 364 (September 18): 1092-1097. 
Sen, Amartya. 1985. Commodities and Capabilities. Amsterdam: Elsevier Science Publishers.

Sen, Amartya. 1988. The Standard of Living. In: The Standard of Living, hrsg. G Hawthorn, 1-38. Cambridge: Cambridge University Press.

Sen, Amartya. 1993. Capability and Well-Being. In: The Quality of Life, hrsg. Martha Nussbaum und Amartya Sen, 30-53. Oxford: Clarendon Press.

Sen, Amartya. 1999. Development as Freedom. New York: Random Books.

Sen, Amartya. 2006. Why Health Equity? In: Public health, ethics, and equity, hrsg. Sudhir Anand, Fabienne Peter und Amartya Sen, 21-33. Oxford: Oxford University Press.

Shiell, Alan. 2007. In Search of Social Value. International Journal of Public Health 52 (6): 333-334.

Siegrist, Johannes und Michael Marmot. 2008. Soziale Ungleichheit und Gesundheit: Erklärungsansätze und gesundheitspolitische Folgerungen. Bern: Huber.

Spencer, Brenda, Ursel Broesskamp-Stone, Brigitte Ruckstuhl, et al. 2008. Modelling the results of health promotion activities in Switzerland: development of the Swiss Model for Outcome Classification in Health Promotion and Prevention. Health Promotion International 23 (1): 86-97.

Sperlich, Stefanie und Andreas Mielck. 2003. Sozialepidemiologische Erklärungsansätze im Spannungsfeld zwischen Schicht-und Lebensstilkonzeptionen. Plädoyer für eine integrative Betrachtung auf der Grundlage der Bourdieuschen Habitustheorie. International Journal of Public Health 11 (2): 165-179.

Stewart, Frances. 2005. Groups and Capabilities. Journal of Human Development 6 (2): 185-204.

Thrane, Christer. 2006. Explaining educational-related inequalities in bealth: Mediation and moderator models. Social Science \& Medicine 62 (2): 467-478.

Veenstra, Gerry. 2007. Social Space, Social Class and Bourdieu: Health Inequalities in British Columbia, Canada. Health \& Place 13 (1): 14-31.

Whitehead, Margaret und Göran Dahlgren. 2006. Levelling up (part 1): a discussion paper on concepts and principles for tackling social inequities in health. Copenhagen: WHO Regional Office for Europe.

Whitelaw, Sandy, Anna Baxendale, Carol Bryce, et al. 2001. Settings' based health promotion: a review. Health Promotion International 16 (4): 339-353.

Williams, Simon J. 1995. Theorising class, health and lifestyles: can Bourdieu help us? Sociology of Health \& Illness 17 (5): 577-604.

Prof. Dr. Thomas Abel, PhD. Universität Bern. Professor für Gesundheitsforschung und Leiter der gleichnamigen Abteilung am Institut für Sozial- und Präventivmedizin. Arbeitsschwerpunkte: Theorie und Empirie der sozialen Differenzierung; Gesundheitsrelevante Lebensstile; Kulturelles Kapital. Adresse: Department of Social and Preventive Medicine, Universität Bern, Niesenweg 6, 3012 Bern, Schweiz; E-Mail: abel@.ispm.unibe.ch

Lic. phil. Dominik Schori, Universität Bern. Wissenschaftlicher Mitarbeiter am Institut für Sozial- und Präventivmedizin. Forschungsschwerpunkte: Soziale Ungleichheit, Capability-Ansatz, Theorie der Praxis. Adresse: Department of Social and Preventive Medicine, Universität Bern, Niesenweg 6, 3012 Bern, Schweiz; E-Mail: dschori@ispm.unibe.ch 


\section{ÖSTERREICHISCHE ZEITSCHRIFT FÜR SOZIOLOGIE

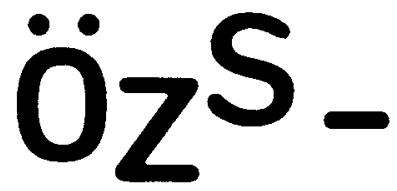

\section{SOZIOLOGIE DER GESUNDHEITSGESELLSCHAFT}

Karl Krajic, Rudolf Forster, Elisabeth Mixa

Editorial. Die „Gesundheitsgesellschaft “ - eine Herausforderung für eine theoretisch orientierte Gesundheits- und Medizinsoziologie? Eine Einleitung Monica Greco

Thinking beyond Polemics: Approaching the Health Society through Foucault

Ausdifferenzierung von spezifischen Funktionssystemen für Krankenbehandlung und Gesundheitsförderung oder: Leben wir in der "Gesundheitsgesellschaft"?

Thomas Abel, Dominik Schori Der Capability-Ansatz in der Gesundheitsförderung: Ansatzpunkte für eine Neuausrichtung der Ungleichheitsforschung

Regina Brunnett Zum Mehrwert von Gesundheit im Postfordismus Milena D. Bister "Jemand kommt zu Dir und sagt bitte": Eine empirische Studie zur Gewebespende im Krankenhauskontext

Robert Grieblet, Wolfgang Dür, Waldemar Kremser Schulqualität, Schulerfolg und Gesundheit. Ergebnisse aus der österreichischen

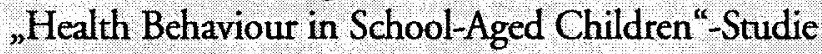

